\title{
Design, Dissemination, and Disinformation in Viral Maps
}

\author{
Anthony C. Robinson \\ GeoVISTA Center, Department of Geography, The Pennsylvania State University
}

Keywords: Virality, map design, machine learning, social media, visualization

Social media has made it possible for maps to reach massive audiences outside of traditional media sources. In some cases, social media maps are original designs crafted by users, in other cases they are modified or replicated from previous sources. It is now relatively easy for novice Internet users to create new maps or manipulate existing images, and social media provides a vehicle for these maps to become visible in ways that were simply not possible even a decade ago. In addition, traditional media sources now harvest content from social streams, and in some cases may amplify what was originally a socially-shared map.

Maps that rapidly reach popularity via social media can be considered viral maps. A key element of virality in social media is the structure of how content becomes viral. The concept of structural virality suggests that the nature of how media are shared is more important than the raw population that might see something (Goel, Anderson et al. 2016). For example, a social media user with millions of followers can broadcast their content to a large audience, but structurally viral content is media that does not require a major broadcaster in order to reach a large audience.

Previous work on viral cartography has shown how viral maps may develop conditions in which their audiences begin creating and repurposing maps in response, resulting in large collections of social media maps. For example, Robinson (Robinson 2018) showed how a viral election map resulted in hundreds of maps shared by social media users in response to the original work.

Viral maps and the maps that emerge in subsequent responses from social media users pose interesting challenges for cartographers to address. Understanding their design dimensions and the ways in which these maps are disseminated (often outside of the social media stream where they may have originated) are two key areas of potential research inquiry. Knowledge of design and dissemination in social mapping is necessary as well if we wish to understand the capability of social media maps to inform or actively disinform the public. We argue that the latter topic is of utmost importance given the relative ease of making maps today versus their clear rhetorical power in public discussion and debate.

New methods are emerging to characterize the design elements of social media maps and their context on the internet. For example, proprietary machine learning services such as Google Cloud Vison and Amazon Rekognition are used for real-time detection of faces, text, sentiment, image structure, and relevant web results. While the primary use case for these services is to support image moderation on social media, to improve search results, and to support marketing activities, these methods can also be applied to the study of social media maps in support of cartographic research.
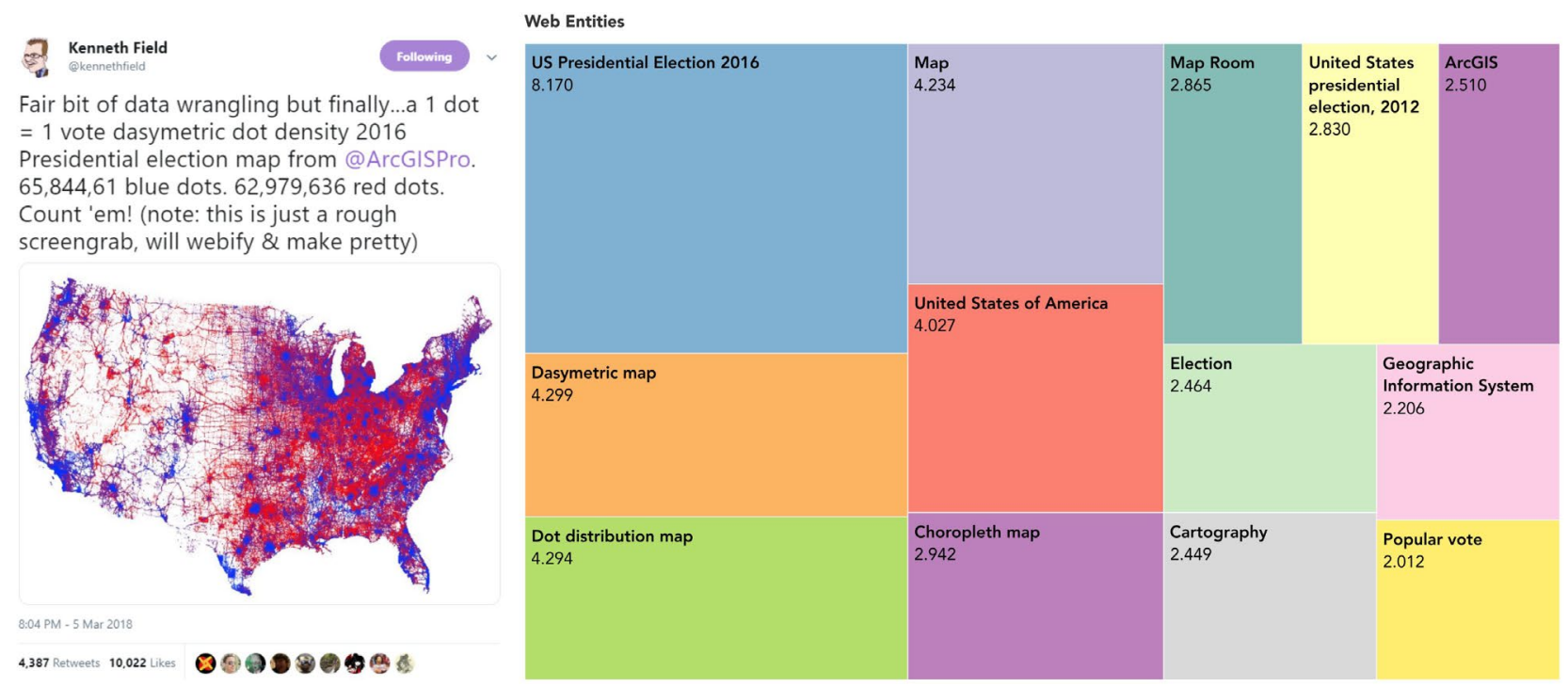

Figure 1. Viral election map designed by Kenneth Field (https://twitter.com/kennethfield/status/970827334038237184) and a treemap visualizing the web entities that are most relevant to this map according to Google Cloud Vision. 
For example, we have used Google Cloud Vision to characterize the design and dissemination of a viral map created and shared by Kenneth Field, a cartographer at Esri. In March of 2018, Field tweeted an image of a dot-density map showing the 2016 United States Presidential Election results. A unique aspect of this map was its ability to show one dot for each of the more than 60 million votes cast in the 2016 election. Field's tweet was liked more than 10,000 times and retweeted over 4000 times, reaching millions of potential viewers.

Google Cloud Vision analysis of Field's map highlights a range of election and cartographic entities that it finds relevant to the original posting (Figure 1). Field's map generated website content that focused on both its meaning in terms of interpreting the 2016 election, as well as its technical execution in terms of cartography. It could be argued that these are not terribly surprising results, but this demonstrates nevertheless that an automated routine has the power to deliver sensible contextual information about map images. Extrapolating from one map to the millions that appear each year on social media, it becomes plausible then to apply machine learning methods to characterize their design and web context, even from streaming sources, as these methods are already built to support real-time analysis of streaming data.

The dissemination of a viral map can be characterized by the number of engagements via social means in both direct and indirect forms. Direct forms of engagement may include user actions to like, share, or reply directly to a social media post. Indirect types of engagement can include the number of people who saw an item in their social media feed, and the potential audience who may have the opportunity to see an item in their social media feeds. In addition, viral maps can become the focus of media attention from traditional news sources, and amplified further to their respective audiences. Finally, users may blog about a viral map or share them in private messages or group chats.

One way to understand the dissemination of a viral map is to take advantage of image analysis service capabilities to produce URLs that show full and partially matching versions of an image. Google Cloud Vision provides this capability along with its other image analysis functions. In the case of the Field dot density map of the 2016 election, webpages that reference the exact image from Field's original tweet include media stories about his map, blog postings, ecommerce sites that sell printed versions of the map, and message forum discussions that reference the map. Partial image matching results reveal only a few sites that have derived versions of Field's original maps, and all of those we reviewed were simply resampled versions of the original. Other partial image matching results included other types of dasymetric and thematic maps located on the web. For example, multiple cellular phone coverage maps are highlighted as partial matches to Field's original work (Figure 2).
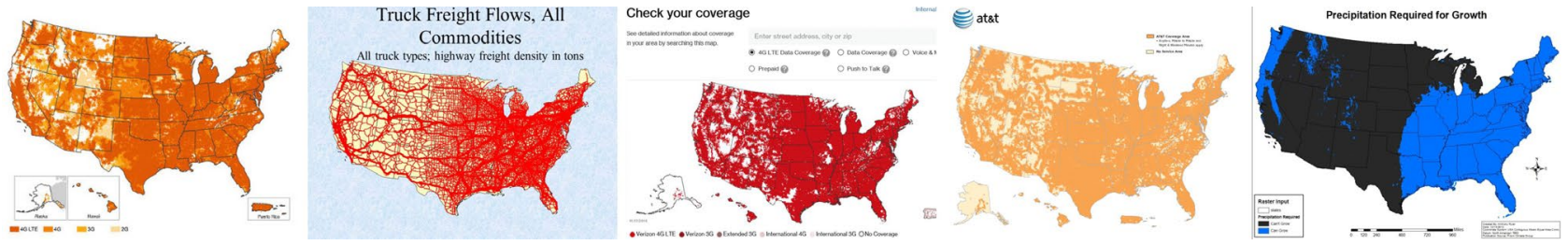

Figure 2. Examples of partial image matches to Field's original viral map as detected by Google Cloud Vision.

We hypothesize that there is considerable potential for social media maps to be sources of disinformation. Map remain a powerful means of communication, and it is easier than ever to create a new map or modify an existing map to convey misleading information. Future research may be able to leverage the attributes and links derived from machine learning image analysis services such as Google Cloud Vision to assess the potential for a viral map to be an agent of disinformation. For example, being able to quickly identify the original source for a map image and to characterize the constellation of websites on which it has been shared may aid users in evaluating the credibility of what they are seeing.

In November 2018, climate scientist Brian Brettschneider shared a map on Twitter that purported to show regions of the United States and their preferred Thanksgiving pie. This map went viral, drawing attention from traditional media sources as well as Twitter users with large audiences of their own, including one U.S. Senator. Many who saw this absurd map argued about its content because they incorrectly assumed it was based on real data. Brettschneider reflected on the power of creating and sharing fake viral maps in a subsequent article for Forbes (Brettschneider 2018), stating, "We cannot let maps, as a medium for communicating information, be co-opted by people with nefarious intentions. I pledge to do my part by clearly noting if a map is a parody in the future."

Brettschneider, B. (2018). "Lessons From Posting A Fake Map." Forbes Retrieved December 5, 2018, from https:/www.forbes.com/sites/brianbrettschneider/2018/11/23/lessons-from-posting-a-fake-map/.

Goel, S., A. Anderson, J. Hofman and D. J. Watts (2016). "The Structural Virality of Online Diffusion." Management Science 62(1): 180-196 DOI: 10.1287/mnsc.2015.2158.

Robinson, A. C. (2018). "Elements of viral cartography." Cartography and Geographic Information Science: 1-18 DOI: 10.1080/15230406.2018.1484304. 